\title{
Interactive comment on "Selection of multi-model ensemble of GCMs for the simulation of precipitation based on spatial assessment metrics" by K. Ahmed et al.
}

\author{
J.H. SUNG. Sung \\ jhsung0524@gmail.com
}

Received and published: 29 May 2019

This manuscript was written fairly well in terms of its academic originality, and scientific descriptions for introduction, methodology, results and conclusions. Especially, it developed a systematic selection framework for many GCMs from CMIP5 based on various state-of-the-art spatial performance metrics. The selected GCMs showed their capability to mimic the spatial patterns of annual and seasonal precipitation. The most impressive point is to summarize so many relevant articles which were published in recent years. This manuscript is worthwhile to be published in this journal. Nevertheless, the following point should be thought carefully in my opinion. This manuscript focuses 
on the simulated data for the very past period (1961-2005). However, the superiority of performances of GCMs for the past period doesn't guarantee the exactness of projection for the future period. In addition, the main objective of GCMs is to support the forecasted future data for 2010-2100. Of course, a part of them can be evaluated using the recent data (2010-2018). If you cannot quantify GCMs' performances for the recent data, you can mention that point in the manuscript. In section 3.3, the four top ranked GCMs were used to generate the most appropriate ensemble of GCMs. Is there any reason why four is used? You can compare your results with those form different numbers of GCMs. This number can affect the results. In section 3.4, you mentioned numerous approaches have been used to calculate the mean time series from an ensemble of better performing GCMs. Thus, it is better to add the reason why two representative methods should be used. What is the improved one? If the method is very critical to the results, you should add the descriptions on simple mean and random forest methods.

Miscellaneous errors When the abbreviation was defined, it should be done at the first appearance. E.g.) p3 L13, root mean square error, p2 L17 multi-model ensemble Check the abbreviation. When any was defined, abbreviation should be used afterwards. E.g. P4 L12, P5 L31 MME, P6 L24 SPAtial EFficiency metric; P6 L8, P10 L7, P16 L24 Rating metric, P11 L5, P15 L19 simple mean and random forest. P7 L12 "lamba"? Check the name of variables in all equations. "N"s in Eq. (5) and (6) are the same? Check the other variables. "(MME)" in the sub-title can be removed. P2 L17 Check Pour et al.(2018b) which is not included in the reference. P2 L22 Check Wang et al.(2017b) which is not included in the reference list. P2 L23 Check Wang et al.(2017a) which is not included in the reference list. P21 L15-16 Salman et al.(2018a) is the same to Salman et al. (2018b), P2 L30 Check Pour et al.(2018b) which is not included in the reference. P3 L8 Are Tebaldi et al. (2005) and Chandler (2013) included in the reference list? P4 L9 "and" should be added at the end of this sentence. P7, P8, P10 Variables " $m$ " and " $n$ " were used in the different equations. Check their consistency. P7 L1 in equation1, is "KGE" correct? SPAEF? P9 L15-16: it should be

Printer-friendly version

Discussion paper 
moved below Equation 8. P9 L17-18: it should be moved below equation 9 and 10. In the conclusions, abbreviations were defined again. Is it correct in this journal? Check HESSD it. P22 L18 Wang et al. (2016) is not cited in the manuscript. Check the reference format.

Grammar P2 L24 "to use" is right? P2 L30, "selection modelling." is right? P3 L7 "such as" was repeated. P3 L25 "scale" or "scales"? P3 L29 "should able" or "should be able"? P5 L16 the second "20" is not necessary. It was already mentioned at the previous sentence. P5 L30 "are" or "is"? P14 L14 "point" or "points"? P14 L18 "scatter" is right? P14 L16 "skillful"? P14 L17 Check the location of"also".

Interactive comment on Hydrol. Earth Syst. Sci. Discuss., https://doi.org/10.5194/hess-2018585, 2019. 